\title{
Male partner involvement in prevention of mother to child transmission of HIV in sub-Saharan Africa: Successes, challenges and way forward
}

\author{
Fatch W. Kalembo ${ }^{1,2^{*}}$, Du Yukai ${ }^{1}$, Maggie Zgambo ${ }^{3}$, Qiu Jun ${ }^{1}$ \\ ${ }^{1}$ Maternal and Child Health Department, Tongji Medical College, Huazhong University of Science and Technology, Wuhan, China; \\ duyukai100@yahoo.com.cn, qiujuntrevor@yahoo.com.cn \\ ${ }^{2}$ Faculty of Health Sciences, Mzuzu University, Mzuzu, Malawi; ${ }^{*}$ Corresponding Author: kalembofatch@yahoo.com \\ ${ }^{3}$ University of North Carolina Project, Lilongwe, Malawi; zgambo.maggie@yahoo.ca
}

Received 3 October 2011; revised 14 November 2011; accepted 27 December 2011

\begin{abstract}
Sub-Saharan Africa has the highest number of mother to child transmissions of HIV. PMTCT programme plays a big role in reducing the MTCT nevertheless its effectiveness in sub-Saharan Africa depends on involvement of male partners considering the fact that men are decision makers in African families. They make important decisions that have big impact on women's health. Male partner involvement has been seen to increase uptake of PMTCT services and their involvement underscores their importance in reducing HIV infection in children. Recently many sub-Saharan countries adopted male partner involvement in PMTCT programme with an aim of increasing the uptake of PMTCT services. The programme has made some progress in improving the effectiveness of PMTCT services. On the other hand the strategy is facing a lot of challenges, the biggest being low male partner involvement. This article therefore seeks to review the successes and challenges faced by male involvement in sub-Saharan Africa. It also proposes the way forward in order to improve its effectiveness. We used peer reviewed articles of research studies conducted in sub-Saharan Africa and other related reliable sources of data to write the paper.
\end{abstract}

Keywords: Male Partner; Involvement; PMTCT; HIVIAIDS; MTCT; Successes; Challenges

\section{INTRODUCTION}

HIV still remains a major challenge globally despite decades of advocacy, awareness raising and investing in pro- grammes to control the spread of HIV. UNAIDS estimated that by $2009,33.3$ million people globally were living with HIV of which 22.5 million were from sub-Saharan Africa. UNAIDS also estimated that within the same period, about 2.5 children globally were living with HIV of which 1.8 million were from sub-Saharan Africa [1]. In sub-Saharan Africa an estimated $60 \%$ of people living with HIV are women, mostly in the reproductive age group. Each year approximately 1.4 million women living with HIV become pregnant. Among antenatal clients in sub-Saharan Africa, the proportion of women living with HIV ranges from $5 \%$ to as high as $30 \%$ and HIV among childbearing women is the main cause of infection among children [2]. Mother-to-child transmission of HIV has been virtually eliminated in industrialized countries, but remains common in Africa. In 2008, for every one child living with HIV in North America and Western and Central Europe, there were nearly 800 children infected with HIV in sub-Saharan Africa [3].

Transmission of HIV from mother to child can take place during pregnancy, labour and delivery as well as after birth via breastfeeding especially mixed feeding. The risk of transmission varies at different stages ranging from $5 \%$ $10 \%$ during pregnancy, $10 \%-20 \%$ during labour and delivery, and $10 \%-20 \%$ through mixed infant feeding. It is estimated that in the absence of any intervention to prevent mother to child transmission, (MTCT) ranges from $15 \%-45 \%$. This rate can be reduced to levels below $5 \%$ with effective interventions [4]. PMTCT was introduced as a comprehensive package of interventions known as Prevention of mother to child transmission (PMTCT) programme with an aim of reducing MTCT. PMTCT programme consists of a range of interventions, including improved antenatal services, opt-out HIV counseling and testing for pregnant women, antiretroviral drug prophylaxis for HIV positive pregnant women and newborns, referral to support groups, and counseling on options for 
safer infant feeding practices. Comprehensive PMTCT programme also includes continued follow-up and treatment for HIV positive mothers and their children, especially for the first 18 months of the child's life [4,5].

The 1994 international conference on Population and development in Cairo and the 1995 international conference on women in Beijing organized by UN called global attention to the importance of involving men in reproductive health programs because of their influence on women's health [6,7]. In 2002 the World Health Organization (WHO) formulated recommendations advising couple HIV testing in settings with high HIV prevalence [8]. WHO's 20102015 PMTCT Strategic Vision, emphasizes the need to involve male partners in scaling up PMTCT services in sub-Saharan Africa. WHO introduced the policy guidelines based on the assumption that couple testing would help increase spousal support for women to use prevention of mother to child transmission (PMTCT) services, create opportunities for secondary prevention by counseling both men and women about HIV, and increase the uptake of testing and identification of HIV infected persons [9].

In PMTCT programme men are encouraged to accompany their pregnant wives to antenatal clinic in order to be counseled and tested for HIV together. Men are also encouraged to be supportive to their pregnant wives if found HIV positive and to encourage them to adhere to all PMTCT protocols. Before the introduction it was observed that a lot of pregnant women were shunning HIV testing because they had no consent from their husbands. Those who had courage to go for test, if tested positive were afraid to disclose their sero status to their husbands because they thought their husbands would accuse them of infidelity or even face divorce. Some HIV positive women who had courage to inform their husbands, faced divorce, violence or accused of infidelity, some were not even allowed to continue with PMTCT interventions. This resulted in low uptake of PMTCT services by HIV positive women. Male partner involvement in PMTCT was then introduced with an aim of combating these problems and consequently increasing uptake and adherence to PMTCT interventions by HIV infected women [5,9].

Since its introduction in sub Saharan Africa, male partner involvement has made an impact in reducing Maternal to child transmission of HIV nevertheless it has its own challenges. This review therefore seeks to establish the impact of male partner involvement in PMTCT in terms of progress made by exploring successes and challenges of the program and suggesting recommendations to improve the programme in sub-Saharan Africa. It uses peer reviewed articles of research studies conducted in subSaharan Africa and other reliable sources of data such as WHO, UNICEF and UNAIDS.

\section{PMTCT PROGRESS IN SUB-SAHARAN AFRICA}

\subsection{HIV Testing and Counseling}

By the end of 2009, six countries in sub-Saharan Africa provided HIV tests to less than one third of all pregnant women: Nigeria (13\%), the Democratic Republic of the Congo (9\%), Ethiopia (16\%), Chad (6\%), Angola (26\%) and Sudan (3\%). In these countries, considerably greater investments are needed to increase HIV testing and counseling among pregnant women in order to effectively prevent mother-to-child transmission of HIV and to enroll eligible women living with HIV in appropriate care and treatment. Four countries reported to have provided HIV testing and counseling to over $80 \%$ of pregnant women in their countries: South Africa (>95\%), Zambia( $>95 \%)$, Botswana (93\%) and Namibia (88\%) [10].

\subsection{Antiretroviral Prophylaxis HIV Pregnant Mothers}

In sub-Saharan Africa, coverage of antiretroviral drugs for preventing mother-to-child transmission reached 54\% in 2009 , from $45 \%$ in 2008 . However, there were considerable differences between sub regions: whereas $68 \%$ of pregnant women received antiretroviral drugs for preventing mother-to-child transmission in Eastern and Southern Africa. In West and Central Africa the comparable figure was $23 \%$. Botswana, Namibia, Swaziland and South Africa (the country with the largest number of pregnant women living with HIV) have already reached the target set at the United Nations General Assembly Special Session (UNGASS) on HIV/AIDS of providing $80 \%$ of pregnant women in need with antiretroviral drugs for reducing the risk of mother-to-child transmission of HIV [10].

The overall gap in reaching the target of $80 \%$ coverage of antiretroviral prophylaxis for preventing mother-to-child transmission is becoming more concentrated in a handful of countries, with 14 countries comprising more than $80 \%$ of the global gap. Nigeria alone now contributes to $32 \%$ of the gap, with the Democratic Republic of the Congo next, contributing $7 \%$ of the gap [11].

\subsection{Antiretroviral Prophylaxis for Infants Born to Mothers Living with HIV}

The 2010 WHO guidelines recommend that all infants born to HIV-positive mothers should receive antiretroviral prophylaxis [10]. About a third of infants in sub-Saharan Africa were reached with antiretroviral prophylaxis (31\% in 2008 and $35 \%$ in 2009). In Eastern and Southern Africa, $45 \%$ of infants received antiretroviral prophylaxis in 2009. Swaziland, Namibia and Botswana, coverage among children was $70 \%$ or higher while in West and Central Africa, only $12 \%$ did. Only Benin and Gambia reached over $40 \%$ of children in need with antiretroviral therapy $[10,11]$. 


\section{CHALLENGES AND OBSTACLES TO SCALE-UP PMTCT IN SUB-SAHARAN AFRICA}

There are a lot of challenges and obstacles to scale up PMTCT services in sub-Saharan Africa. Most PMTCT programs in sub-Saharan Africa focus only on medical intervention, leaving out the others that address the social drivers of MTCT including primary prevention, male involvement, family planning and other reproductive health measures [3]. PMTCT services continue to suffer from low counseling uptake and low testing proportions. Furthermore, loss to follow-up is high, as mothers may either not return for delivery or deliver in a health facility which does not offer a PMTCT service [12].

HIV testing of men also remains challenging, with an estimated $6.1 \%$ of men in sub-Saharan Africa having ever been tested for HIV and receiving the results [13]. Further challenges include large proportions of home deliveries, shortages of personnel, inadequate supplies of test kits, varying distribution and availability of PMTCT service delivery points, lack of supplementary feeds for women who may opt for non-breastfeeding for their infants, and logistical and social implications after testing HIV positive, such as a lack of spousal support and sometimes violence [14].

Some countries have been slow to revise national policy to reflect global guidance on PMTCT and peadiatrics treatment and care, in particular recent guidance that recommends use of a combination of drugs for ARV prophylaxis in pregnant women. Policies are not always disseminated well. Health workers at clinic facilities are often unaware of existing policies and guidelines and unclear about what advice to give to mothers living with HIV about infant feeding. Failure to promote exclusive breastfeeding, unless in circumstances where replacement feeding is acceptable, feasible, affordable, sustainable and safe is also putting infants at increased risk of HIV and of other infections as a result of mixed feeding or early cessation of breastfeeding. There is often a lack of standard operational guidelines to support the implementation of comprehensive PMTCT and ensure that services are integrated. Weak health systems are a significant challenge, in particular shortages of key cadres of health workers, inadequate equipment and poor procurement and supply management. Scale-up is difficult when health services lack staff that can provide HIV counseling and testing, administer ARVs and advise women about family. Follow-up treatment and care for mothers and children is still limited in many countries, Pregnant women may be deterred from finding out their HIV status and accessing PMTCT services in contexts where HIV is highly stigmatized and male involvement is limited. Data on infant feeding choices and the quality of follow up treatment, care and support for women and infants is not available from many of the sub-Saharan Africa countries surveyed $[14,15]$.

\section{MALE PARTNER INVOLVEMENT IN PMTCT IN SUB-SAHARAN AFRICA}

\subsection{Rationale for Male Partner Involvement in PMTCT}

Men are decision makers in many of the African settings where PMTCT is offered [16]. Without working with men change would be very difficult or impossible [17]. Literature shows that risk behaviors change dramatically among couples where partners are aware of their HIV serostatus [18]. One major factor that prevents some women from accepting HIV testing is the need to seek their partner consent or assent [19]. Across sectional study in Uganda found that the strongest predictor of willingness to accept an HIV test was the woman's perception that her husband would approve of her testing for HIV. Women who thought their Husbands would approve were almost six times more likely to report a willingness to be tested compared to those who thought their husbands would not approve $(\mathrm{OR}=5.6,95 \%$ CI $2.8,11.2)[20]$.

Studies in Uganda, Malawi and Nigeria have shown that the utilization of PMTCT services by pregnant women is influenced by individual factors such as fear of disclosure of HIV results, lack of male partner support, fear of violence, abandonment and stigmatization [21-23]. In Cote d'Ivoire, $3.5 \%$ to $14.6 \%$ of pregnant women reported negative consequences of HIV status disclosure to their spouses [24]. In sub-Saharan Africa women's economic vulnerability and dependence on men coupled with traditional male superiority over women increase their vulnerability to HIV by constraining their ability to negotiate the use of a condom, discuss fidelity with partners, or leave risky relationships $[25,26]$. This means that if men are involved in PMTCT their understanding in HIV prevention will be higher and they would cooperate with their spouses in using condoms and other HIV preventive measures. The rate of violence, stigmatization and abandonment can also be reduced. Men always play a leading role in terms of initiating sex. In Malawi, men initiate sex in $92 \%$ of relationships and women feel powerless to refuse sex or negotiate safe sex [5]. Traditionally it is common for men to have extra marital relationships thereby putting their spouses at risk of contracting HIV. A study in Tanzania found that the risk for HIV was greater among women whose male partner had other sexual partners [27]. Another study in Tanzania found that male partner played a role in terms of woman's risk of acquiring HIV [12].

With male partner involvement in PMTCT, a couple has a chance to make informed decisions together on living positively with HIV, share responsibility for preventing 
HIV in the unborn child and they can discuss safer sex practices and make informed decisions to access care and treatment [28]. Men can play an important role of supporting HIV positive pregnant women to get to clinics or hospitals where chances of safe delivery are higher, they can assist HIV positive pregnant women to choose safe infant feeding method [18].

\subsection{Successes of Male Partner Involvement in PMTCT}

In Zambia and Kenya, couple HIV counseling appeared to improve the acceptability of HIV testing, uptake of ARV prophylaxis and adherence to prolonged and mixed breastfeeding. Women who received couple counseling did not report an increased risk of adverse social events compared to individually counseled women [27,30,31]. Dropout among those who have discussed HIV testing with their partners was found to be low in Burkina Faso [32]. A study conducted in Ivory Coast revealed that men, who were involved in PMTCT program played an active role in applying the advice received, particularly related to exclusive breast feeding and early weaning. Studies in Kenya found that women accompanied by their partner for HIV testing were three times more likely to return for antiretroviral prophylaxis. Couple post-test counseling was also associated with an eight-fold increase in postpartum follow up, as well as greater antiretroviral utilization and formula feeding $[30,33]$. In Uganda male involvement is associated with beneficial health outcomes such as first trimester antenatal visits, abstinence from smoking and alcohol consumption, reduction in low birth weight infants as well as positively influence uptake of HIV testing and preventive interventions for vertical and sexual transmissions of HIV [34]. In another study in Kenya the combined adverse outcome risk of vertical transmission and infant mortality was significantly lower with male partner involvement. The combined risk for either vertical transmission or mortality was $45 \%$ lower with male antenatal attendance [35].

\subsection{Challenges of Male Partner Involvement in PMTCT}

Literature shows that there is low male partner involvement in PMTCT services in many sub-Saharan countries $[12,36]$. In sub-Saharan Africa, male participation rate levels in hospital settings vary between $12.5 \%$ and $18.7 \%$ [37]. In Malawi, male partners do not often come forward to test for HIV with their wives, this has contributed to drop outs and non-compliance at many levels of PMTCT services $[28,38,39]$. In Tanzania, male involvement in reproductive and child health services is low, estimated at 5\% and lower in urban areas [40]. Several factors have been found as barriers to male involvement. These include; culture, health system, socio-economic factors, lack of information, poor communication, stigma and lack of confidentiality [38-44].

\subsubsection{Cultural Factors}

Traditionally in sub-Saharan Africa, support and care are seen as women's work [29]. In Tanzania social and religious norms that prohibits males from attending female health services and the wide spread attitude that female reproductive health is not male responsibility was found to inhibit male involvement in PMTCT [40]. In Burkina Faso and Cameroon, Antenatal clinic activities were perceived by many fathers as outside their responsibility $[12,13]$. In Eastern Uganda the power structure of marriages in which men are decision makers and have power over wives' action results to men resisting women's effort to influence them to have HIV testing [36]. A study conducted in four countries; Cameroon, Dominican republic, Georgia and India indicated that male partner rarely participated in antenatal care services, mainly because these are traditionally and programmatically a woman's domain [41]. Another barrier is men's perception that he will be viewed as jealous by the community if he comes to clinic with the pregnant wife [13]. In Malawi, in settings where a man moves to a woman's village when married, revelation of HIV positive diagnosis by his wife leads to a husband being looked upon as the one who brought the infection to his wife's clan and kin. The experience of blame for infecting the family of his in-laws will commonly be so difficult to live with that husband eventually leaves his wife and children to manage on their own. This has led to PMTCT programme being locally named "the divorce programme" [42].

\subsubsection{Socio-Economic Factors}

In Malawi, Men reported that due to socio-economic difficulties, they did not have time to attend ANC with their partners since they utilize the time to source money to take care of their families [43]. In South Africa and Uganda, distance, poor roads, undeveloped transport system and cost of getting to the hospital bar men from being involved in PMTCT since most of them have few resources to travel and live along distance from the clinic or hospital $[36,44]$. In Uganda, charging user fee also prevent male partner from participating in PMTCT services due to financial problems $[21,36]$.

\subsubsection{Health System Factors}

In Uganda, men reported having been forced to wait an entire day for care at antenatal clinics, a heavy sacrifice for someone who needs to work to support his family. They were also excluded from the session where their wives were examined and had to wait outside without any information about what was happening to their pregnant wives. Furthermore, health workers mistreatment of the spou- 
ses made them feel uncomfortable and embarrassed $[21,36]$. Lack of adequate space in the antenatal clinics coupled with shortage of health workers and an increase in women attending antenatal care demotivate men from attending ANC with their spouses since they have to wait for a long time before they are attended to [21].

\subsubsection{Lack of Information, Trust and Poor Communication}

Lack of information on PMTCT and HIV testing in Tanzania has contributed to low male partner involvement in PMTCT services [40]. Poor communication between spouses on PMTCT has also contributed to low turnout of men in antenatal clinics in sub-Saharan Africa [45]. In a study conducted in Cameroon most women conceded that they avoid talking about safe sex and HIV/AIDS to their partner because they feel embarrassed if they start conversation on these issues. Some even fear that their partners would accuse them of infidelity [41].

\subsubsection{Stigma and Lack of Confidentiality}

In a study conducted in rural western Uganda, AIDS related stigma and lack of confidentiality created barriers to seeking VCT among men. More than half of the men feared to test for HIV because of stigma. Men were worried of being labeled HIV-infected because they would lose their social privileges. They expressed fear of meeting familiar people in HIV testing clinics, and preferred to test in distant clinics where they were not known by the people and staff [46].

\subsection{Way Forward in Male Partner Involvement in PMTCT}

Innovative approaches to promote male partner involvement are urgently needed in order to enhance uptake of PMTCT interventions [47]. Literature has suggested several strategies to improve male involvement in PMTCT. Tanzania showed a $30 \%$ increase in male partner counseling when men were sent a letter of invitation to participate in PMTCT programs $[12,48,49]$. Other strategies include extending clinic hours so that men who have tight work or business schedule can visit in late afternoon. Reducing wait times for men or couples who visit $\mathrm{MCH}$ clinics in order to give chance to men to return to work and business in time. Health workers should attend ward development and other community meetings to explain the importance of male involvement and the need for expectant parents to be tested $[48,50]$.

PMTCT program should integrate beliefs, values and practices of different cultural settings so that it can attract more male partners [42]. Communication with partner plays a vital role in the uptake of HIV testing. Encouraging women to engage in discussion about testing with their part- ners may improve male partner participation in PMTCT [21]. Refresher courses for midwives and nurses should be conducted on regular basis so that they can make PMTCT services more male friendly. Other studies suggested, Weekend clinics hours, making clinics more male or couple friendly or introducing specific clinics for pregnant couples as effective ways to increase male involvement in PMTCT activities $[12,49]$. Governments should also bring services closer to the people for easy access. A three-arm randomized controlled trial (RCT) was conducted in DRC that offered VCT at a neighborhood health center, bar and church to male partners of pregnant women attending a maternity unit in Kinshasa. Male participation was significantly higher in bars $(26 \%, \mathrm{P}, 0.001)$, and higher but not statistically significant in church-based VCT (21\%, P $1 / 40.163)$ compared with health centre VCT (18\%). Male participation in VCT associated with ANCs was higher in non-health service settings, particularly in bars [37].

Welfare of health workers should be improved so that they are motivated to carry out their duties whole heartedly in addition to this more staff should be recruited in the health service so that waiting time for ANC attendees is reduced [21]. In South Africa, Uganda and Burkina Faso, use of male nurses to motivate male partners and the use of IEC in the community to encourage men to accompany their wives to ANC was found to be useful [30,32,34,52]. Peer influence and role models, provision of transport to testing site, provision testing to non-governmental organization and provision of home-based testing were also found to be effective in improving male involvement in PMTCT [36]. In Democratic Republic of Congo at Kingasani maternity, male participation in PMTCT was increased from $2 \%$ to $18 \%$ when men were invited to PMTCT services between 15:00 and 20:00 hours through letters [37]. A study in Tanzania where men were asked on their approval of different ways to overcome men's barriers, the highest affirmation was expressed for special couple voluntary counseling and testing (CVCT) hours within ANC services (91 persons, $73 \%$ ), followed by a special waiting area for men/couples ( 75 persons, $61 \%$ ), an invitation letter from the health facility delivered to men by their wives ( 58 persons, $47 \%$ ) and an official letter excusing from work for ANC attendance (53 persons, $43 \%$ ). 80 interviewees (65\%) suggested to offer CVCT in ANC services not only during working days to enable male partners to attend [51].

\section{CONCLUSION AND PERSPECTIVES}

Men are decision makers in many societies and families in sub-Saharan Africa; they make important decisions that affect the health of the family members positively or negatively. Literature has shown that male partner involvement in PMTCT is very important for successful imple- 
mentation of the programme. It can increase up take of PMTCT services by HIV positive pregnant women. Literature has also shown that countries which are doing well in PMTCT programme already adopted male partner involvement. The biggest challenge being faced by many countries is low participation of male partners in PMTCT services. Lack (or low) of male involvement implies low uptake of PMTCT interventions and increase in maternal to child transmission of HIV. There is need for more research studies in sub-Saharan countries to find innovative strategies that do not only consider male partners as mere supporters of women in PMTCT services but rather as active participants. Such strategies should also incorporate ways of changing the mind set of African men of perceiving motherhood and PMTCT as women's domain but rather as a collective responsibility. Some countries in sub-Saharan Africa are silent on the role of male partners in PMTCT. There is need for research studies in such countries to explore the feasibility and importance of male partner involvement in PMTCT services. Few research studies have also been done to establish the rate of male involvement in PMTCT in sub-Saharan Africa. There is need for further research on this area in order to determine the level of male partner involvement in PMTCT which can be of great importance in planning for PMTCT scale-up activities.

\section{REFERENCES}

[1] UNAIDS (2010) Report on the global AIDS epidemic. http://www.unaids.org/global report/globalreport.htm

[2] UNAIDS (2010) Africa prepares to eliminate mother-tochild transmission of HIV by 2015, 26 May.

http://www.unaids.org/en/resources/presscentre/featuresto ries/2010/may/20100526pmtct

[3] UNFPA, WHO, UNAIDS (2010) It takes a village ending mother-to-child HIV transmission, a partnership uniting the Millennium Villages Project and UNAIDS.

http://www.unaids.org/en/media/unaids/contentassets/doc uments/document/2010/20101231 MVP en.pdf

[4] WHO (2011) Mother to child transmission of HIV. http://www.who.int/hiv/topics/mtct/en/index.html

[5] Malawi Ministry of Health (2007) PMTCT training module.

[6] United Nations Population Information Network (1994) Proceedings of International Conference on Population and Development, Cairo, 2004. http://www.un.org/popin/icpd2.htm

[7] United Nations Division for the Advancement of Women (1995) Proceedings of Fourth World Conference on Women, Beijing. http://www.un.org/womenwatch/daw/beijing/platform

[8] WHO (2002) Increasing access to HIV testing and counseling: Report of a WHO consultation, 19-21 November 2002, Switzerland. Geneva W.C.o.I.A.t.H. Testing and a.
Counseling.

[9] WHO (2010) PMTCT strategic vision 2010-2015. http://www.who.int/hiv/pub/mtct/strategic_vision/en/inde x.html

[10] WHO Progress Report (2010) Scaling up HIV services for women and children. http://www.who.int/hiv/topics/mtct/data/en/index3.html

[11] UNAIDS (2010) Report on the global AIDS epidemic. http://www.unaids.org/documents/20101123 GlobalRepo rt em.pdf

[12] Pignatelli, et al. (2006) Factors predicting uptake of voluntary counseling and testing in a real-life setting in a mother-and-child center in Ouagadougou, Burkina Faso. Tropical Medicine and International Health, 11, 350-357. doi:10.1111/j.1365-3156.2006.01564.x

[13] Nkuoh, G.N., Meyer, D.J., Tih, P.M. and Nkfusai, J. (2010) Barriers to men's participation in antenatal and prevention of mother-to-child HIV transmission care in Cameroon Africa. Journal of Midwifery, Women's Health, 55, 4. doi:10.1016/j.jmwh.2010.02.009

[14] Kasenga, F., Byass, P., Emmelin, M. and Hurting, A. (2010) The implications of policy changes on the uptake of a PMTCT programme in rural Malawi: First three years of experience. Global Health Action, 2, 10. doi:10.3402/gha.v3i0.1882

[15] Tear Fund (2011) Scaling up prevention of motherto-child transmission of HIV. http://www.tearfund.org

[16] Akarro, R., Deonisia, M. and Sichona, F. (2011) An evaluation of male involvement on the programme for PMTCT of HIV/AIDS: A case study of Ilala Municipal in Dares Salam, Tanzania. Arts and Social Science Journal, ASSJ, 20.

[17] Sternberg, P. and Hubley, J. (2004) Evaluating men's involvement as a strategy in sexual and reproductive health promotion. Health Promotion International, 19, 389-396. doi:10.1093/heapro/dah312

[18] Medley, M., Moreno, C., McGill, S. and Maman, S. (2004) Rates, barriers and outcomes of sero status disclosure among women in developing countries: Implications for prevention of mother to child transmission programmes. Bulletin of the World Health Organization, 82, 299-307.

[19] Omatayo, et al. (2007) Approaches for scaling up human immunodeficiency virus testing and counseling in prevention of mother to child human immunodeficiency virus transmission settings in resource limited countries. American Journal of Obstetrics and Gynecology, supplement to September S81-S88. doi:10.1016/j.ajog.2007.03.006

[20] Bajunirwe, F. and Muzoora, M. (2005) Barriers to the implementation of programs for the prevention of mother-to-child transmission of HIV: A cross-sectional survey in rural and urban Uganda. AIDS Research and Therapy, 2, 10. doi:10.1186/1742-6405-2-10

[21] Byamugisha, R., Tumwine, J.K., Semiyaga, N. and Tylleskar, T. (2010) Determinants of male involvement in the prevention of mother-to-child transmission of HIV programme in Eastern Uganda: A cross-sectional survey. Re- 
productive Health, 7, 12. doi:10.1186/1742-4755-7-12

[22] Van Lettow, et al. (2011) Uptake and outcomes of prevention of mother to child transmission (PMTCT) program in Zomba district Malawi. BMC Public Health, 11, 426. doi:10.1186/1471-2458-11-456

[23] Brou, et al. (2007) When do HIV-infected women disclose their HIV status to their male partner and why? A study in PMTCT programme, Abidjan. PloS Medicine, 4, 12. doi:10.1371/journal.pmed.0040342

[24] Brou, H., Agbo, H. and Du-Lou, G. (2005) Impact of HIV counseling and testing during antenatal consultation for HIV-women in Abidjan (Cote d'Ivoire): A quantitative and qualitative study [in French]. Sante, 15, 81-91.

[25] Ramasundaram, S. (2002) How men's power over women fuels the HIV epidemic; It limits women's ability to control sexual interactions. $B M J, 1,324$.

[26] Peacock, D., Stemple, L., Sawires, S. and Coates, T.J. (2009) "Men make a difference" campaign launched in 2000 by the United Nations to engage men in HIV prevention. Men, HIV and human rights. Journal of Acquired Immune Deficiency Syndromes, 3, 119-125. doi:10.1097/QAI.0b013e3181aafd8a

[27] Orne-gliemann, et al. (2010)Couple-oriented prenatal HIV counseling for HIV primary prevention: An acceptability study Cameroon, Dominican Republic, George and India. BMC Public Health, 10, 197. doi:10.1186/1471-2458-10-19

[28] Malawi, Ministry of Health (2008) PMTCT of HIV and peadiatric HIV care guidelines. 2nd Edition, Ministry of Health, Lilongwe.

[29] Peacock, D. (2003) Men as partners: Promoting men's involvement in care and support activities for people living with HIV/AIDS. United Nations Development Programme (UNDP) Proceedings of Expert Group Meeting on "The Role of Men and Boys in Achieving Gender Equality", Brasilia, 21-24 October 2003.

[30] Betancourt, T.S., Abrams, E.J., McBain, R. and Fawzi, M.S. (2010) Family centred approaches to the prevention of mother-to-child transmission of HIV. Journal of the International AIDS Society, 13, S2-S11. doi:10.1186/1758-2652-13-S2-S2

[31] Semrau, et al. (2005) Women in couples antenatal HIV counseling and testing are not more likely to report adverse social events. AIDS, 19, 603-609.

[32] Sarker, M., Sanou, A., Snow, R., Ganame, J. and Gondos, A. (2007) Determinants of HIV counseling and testing participation in a prevention of mother-to-child transmission programme in rural Burkina Faso. Tropical Medicine and International Health, 12, 1475-1483. doi:10.1111/j.1365-3156.2007.01956.x

[33] Farquhar, et al. (2004) Antenatal couple counseling increases uptake of intervention to prevent HIV-1 transmission. Journal of Acquired Immune Deficiency Syndromes, 37, 1620-1626. doi:10.1097/00126334-200412150-00016

[34] Tweheyo, R., Konde-Lule, J., Tumwesigye, N.M. and Sekandi, J.N. (2010) Male partner attendance of skilled antenatal care in peri-urban Gulu district, northern Uganda. Pregnancy and Child Birth, 10, 53. doi:10.1186/1471-2393-10-53

[35] Aluisioet, et al. (2011) Male antenatal attendance and HIV testing are associated with decreased infant HIV infection and increased HIV-free survival in Nairobi, Kenya. Journal of Acquired Immune Deficiency Syndromes, 56, 76-82. doi:10.1097/QAI.0b013e3181fdb4c4

[36] Larsson, et al. (2010) Mistrust in marriage-Reasons why men do not accept couple HIV testing during antenatal care-A qualitative study in eastern Uganda. $B M C$ Public Health, 10, 769. doi:10.1186/1471-2458-10-769

[37] Ditekemena, et al. (2011) Male partner voluntary counseling and testing associated with the antenatal services in Kinshasa, Democratic Republic of Congo: A randomized controlled trial. International Journal of STD \& AIDS, 22, 165-170. doi:10.1258/ijisa.2010.010379

[38] Murphy, P.J. (2007) Health care providers' perspectives on the barriers to prevention of mother-to-child HIV transmission in Thyolo, Malawi. Centre for Global Initiatives, Carolina Papers on International Health, 24. http://gi.unc.edu/research/carolina-papers/health-papers.h $\underline{\mathrm{tml}}$

[39] Chinkonde, J.R., Sundby, J. and Martison, F. (2009) The prevention of mother-to-child HIV transmission programme in Lilongwe, Malawi: Why do so many women drop out. Reproductive Health Matters, 17, 143-151. doi:10.1016/S0968-8080(09)33440-0

[40] Family Health International (2011) Male partnership in PMTCT and antenatal services FHI in Tanzania's Dodoma Region, used strategies to involve men in the services and in HIV testing.

http://www.searchsite.fhi360.org/cgi-bin/MsmGo.exe?gra b id=121350674\&extra arg=\&page id=2997\&host id=2 \&query=PMTCT\&hiword=PMTCT+PMTC +

[41] Pulerwitz, et al. (2010) Addressing gender dynamic and engaging men in HIV programs. Lessons learned from Holizons Research. Public Health Reports, 125.

[42] Njunga, J. and Blystad, A. (2010) The divorce program: Gendered experiences of HIV positive mothers enrolled in PMTCT programs-The case of rural Malawi. International Breastfeeding Journal, 5, 14. doi:10.1186/1746-4358-5-14

[43] Aarnio, et al. (2010) Male involvement in antenatal HIV counseling and testing, exploring men's perceptions in rural Malawi. AIDS, 12, 21.

[44] Skinner, D. Mfecane, S. and Gumende, T. (2005) Barriers to accessing PMTCT services in rural area, South Africa. African Journal of AIDS Research, 2, 5.

[45] Health Bridge (2011) Africa community of practice on primary health care. Challenges in the prevention-ofmother-to-child-transmission of HIV in Africa. http://www.healthbridge.ca

[46] Bwambale, F.M., Ssali, S., Byaruhanga, S., Kalyango, J. and Karamagi, C. (2008) Voluntary HIV counselling and testing among men in rural western Uganda: Implications for HIV prevention. BMC Public Health, 8, 263. doi:10.1186/1471-2458-8-263

[47] Shetty, et al. (2008) The feasibility of prevention of mother-to-child transmission of HIV using peer counsel- 
ors in Zimbabwe. AIDS Research and Therapy, 5, 17. doi:10.1186/1742-6405-5-17

[48] AuVinem, J., Suominen, B. and Valimaki, C. (2010) Male participation and prevention of human immunodeficiency virus HIV, mother-to-child transmission in Africa. Psychology, Health and Medicine, 3, 15.

[49] Kizito, et al. (2008) Uptake of HIV and syphilis testing of pregnant women and their male partners in a programme for prevention of mother to child of HIV in Uganda.
Tropical Medicine and International Health, 13, 680-682. doi:10.1111/j.1365-3156.2008.02052.x

[50] Reece, et al. (2010) Assessing male spousal engagement with prevention of mother to child transmission (PMTCT) programs in western Kenya. AIDS Care, 6, 22. doi:10.1080/09540120903431330

[51] Theuring, et al. (2009) Male involvement in PMTCT services in Mbeya Region, Tanzania. AIDS and Behavior, 13, 92-102. doi:10.1007/s10461-009-9543-0 